\title{
Application of the heart failure meta-score to predict prognosis in patients with cardiac resynchronization defibrillators
}

\author{
Dominic A.M.J. Theuns ${ }^{\mathrm{a}, *}$, Beat A. Schaer ${ }^{\mathrm{b}}$, Kadir Caliskan ${ }^{\mathrm{a}}$, Sanne E. Hoeks ${ }^{\mathrm{c}}$, Christian Sticherling ${ }^{\mathrm{b}}$, \\ Sing-Chien Yap ${ }^{\text {, }}$ Ana Carolina Alba ${ }^{\mathrm{d}}$
}

a Dept. of Cardiology, Erasmus MC, Rotterdam, the Netherlands

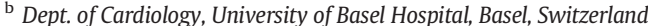

c Dept. of Anesthesiology, Erasmus MC, Rotterdam, the Netherlands

${ }^{\mathrm{d}}$ Heart Failure/Transplant program, Toronto General Hospital, University Health Network, Toronto, Ontario, Canada

\section{A R T I C L E I N F O}

\section{Article history:}

Received 17 October 2020

Received in revised form 23 December 2020

Accepted 3 January 2021

Available online $\mathrm{xxxx}$

\section{Keywords:}

Implantable cardioverter-defibrillator

Cardiac resynchronization therapy

Primary prevention

Risk stratification

Mortality

\begin{abstract}
A B S T R A C T
Background: The Heart Failure (HF) Meta-score may be useful in predicting prognosis in patients with primary prevention cardiac resynchronization defibrillators (CRT-D) considering the competing risk of appropriate defibrillator shock versus mortality.

Methods: Data from 648 consecutive patients from two centers were used for the evaluation of the performance of the HF Meta-score. The primary endpoint was mortality and the secondary endpoint was time to first appropriate implantable cardioverter-defibrillator (ICD) shock or death without prior appropriate ICD shock. Fine-Gray model was used for competing risk regression analysis.

Results: In the entire cohort, 237 patients died over a median follow-up of 5.2 years. Five-year cumulative incidence of mortality ranged from $12 \%$ to $53 \%$, for quintiles 1 through 5 of the HF Meta-score, respectively (logrank $P<0.001$ ). Compared with the lowest quintile, mortality risk was higher in the highest quintile (HR 6.9; 95\%CI 3.7-12.8). The HF Meta-score had excellent calibration, accuracy, and good discrimination in predicting mortality ( $C$-statistic 0.76 at 1 -year and 0.71 at 5 -year). The risk of death without appropriate ICD shock was higher in risk quintile 5 compared to quintile 1 (sub HR 5.8; 95\%CI 3.1-11.0, $P<0.001$ ).

Conclusions: Our study demonstrated a good ability of the HF Meta-score to predict survival in HF patients treated with CRT-D as primary prevention. The HF Meta-score proved to be useful in identifying a subgroup with a significantly poor prognosis despite a CRT-D.
\end{abstract}

(c) 2021 The Authors. Published by Elsevier B.V. This is an open access article under the CC BY license (http:// creativecommons.org/licenses/by/4.0/).

\section{Introduction}

Heart failure (HF) is a progressive disease associated with high morbidity and mortality. The prevalence of HF is increasing with high burden of costs and loss of quality of life [1,2]. Data from randomized clinical trials have shown a benefit of cardiac resynchronization therapy (CRT) in reducing morbidity and mortality in selected patients with drug refractory HF, reduced left ventricular ejection fraction (LVEF), and electrical dyssynchrony [3-6]. As the majority of patients with HF with reduced $\mathrm{EF}$ (HFrEF) have a potential indication for a prophylactic implantable defibrillator (ICD), ICDs combined with CRT (CRT-D) are part of standard management in patients with HFrEF and left bundle branch block $[7,8]$. Despite the beneficial effects of CRT, mortality is not uniform among patients as CRT-candidates have heterogeneous

* Corresponding author at: Department of Cardiology, Erasmus MC, Room Rg-632, PO Box 2040, Rotterdam, CA 3000, The Netherlands.

E-mail address: d.theuns@erasmusmc.nl (D.A.M.J. Theuns). risk profiles. Patients may have mild to severe HF, different etiology of $\mathrm{HF}$, and different burden of various potentially co-existing comorbidities. Several models have been developed to predict mortality risk in patients with HF, such as the Seattle Heart Failure Model (SHFM) and the Heart Failure Survival Score (HFSS) [9,10]. Recently, a novel prediction model derived from a meta-analysis, the HF Meta-score, was developed [11]. The provincial Ontario database was used for validation which included a mixed population of patients with primary and secondary prevention indication treated with ICDs or CRT-Ds, $19 \%$ of whom received a de novo CRT implantation. As a consequence, the performance of this novel prediction model in primary prevention patients with a CRT-D is unknown. The aim of this study was to assess the performance of the HF Meta-score when applied to a real-world cohort of primary prevention patients who underwent CRT-D implantation. In addition, the rationale to implant a CRT-D depends on two mutually exclusive competing events, appropriate ICD shocks and death without prior ICD therapy. Therefore, we also evaluated whether predicted risks by the HF Metascore could identify the subset of patients who die before appropriate ICD shocks. 


\section{Methods}

\subsection{Study population}

We used data from two prospective ICD registries of the cardiology departments of Erasmus MC (Rotterdam, the Netherlands) and the University Hospital of Basel (Basel, Switzerland). In these registries, we identified all patients in whom a CRT-D was implanted for chronic HF and primary prevention of sudden cardiac death (SCD) between January 2005 to December 2017 (Rotterdam cohort n=426; Basel cohort $\mathrm{n}=222$ ). In both cohorts, CRT implantation was indicated by symptomatic HF despite optimal medical therapy, an impaired LVEF ( $\leq 35 \%)$, and the presence of an inter- or intraventricular conduction delay (QRS duration $\geq 130 \mathrm{~ms}$ ). The administrative censoring date was set at the end of December 2018 for all patients alive until that date. Over the years, indications for CRT and programming of devices have changed, in order to identify possible trends, we defined three groups according to the implant year (1: 2005-2009; 2: 2010-2014; 3: 2015-2017).

The study protocol was approved by the IRB of the Erasmus MC (MEC 2018-1713) and the University Hospital of Basel (BASEC 2018329). This retrospective study was not subjected to the Dutch Medical Research Involving Human Subjects Act and the need for written informed consent was waived. The study was carried out according to the ethical principles for medical research involving human subjects established by the Declaration of Helsinki. The privacy of all patients and the confidentiality of their personal information were protected.

\subsection{Data collection}

This study included a total of 648 CRT-D patients. Demographic and clinical data listed in Table 1 were obtained before implantation. Laboratory data were collected the day before implantation. For all

\section{Table 1}

Baseline characteristics of the study cohort $(n=648)$.

\begin{tabular}{ll}
\hline Variable & \\
\hline Age (y) & $66(58-72)$ \\
Male gender & $492(76 \%)$ \\
Atrial fibrillation & $125(19 \%)$ \\
Ischemic cardiomyopathy & $305(47 \%)$ \\
NYHA functional class & \\
II & $219(34 \%)$ \\
III & $407(63 \%)$ \\
IV & $22(3 \%)$ \\
LVEF (\%) & $25 \pm 6$ \\
QRS duration (ms) & $167 \pm 23$ \\
Heart failure admission 1 year prior to implantation & $208(32 \%)$ \\
Comorbidities & \\
Diabetes mellitus & $163(25 \%)$ \\
Chronic obstructive pulmonary disease & $85(13 \%)$ \\
Peripheral vascular disease & $53(8 \%)$ \\
Laboratory data & \\
Sodium (mmol/l) & $139 \pm 4$ \\
Hemoglobin (g/dl) & $13.6 \pm 1.7$ \\
Creatinine (mg/dl) & $1.1(0.9-1.5)$ \\
Glomerular filtration rate $\left(\mathrm{ml} / \mathrm{min} / 1.73 \mathrm{~m}^{2}\right)$ & $64(47-82)$ \\
Glomerular filtration rate $<60 \mathrm{ml} / \mathrm{min} / 1.73 \mathrm{~m}^{2}$ & $294(45 \%)$ \\
Medical therapy & $541(84 \%)$ \\
Beta-blocker & $595(92 \%)$ \\
ACEI/ARB & $328(51 \%)$ \\
MRA & $523(81 \%)$ \\
Diuretic & $125(19 \%)$ \\
Digoxin & $386(60 \%)$ \\
Statin & \\
\hline
\end{tabular}

Continuous data are expressed as mean \pm SD or median (interquartile range), and categorical data as number (percentage).

ACEI, angiotensin-converting enzyme inhibitor; ARB, angiotensin receptor blocker; LVEF, left ventricular ejection fraction; MRA, mineralocorticoid receptor antagonist; NYHA, New York Heart Association. patients, the renal function was assessed by estimating the glomerular filtration rate (eGFR) using the abbreviated Modification of Diet in Renal Disease equation [12]. Impaired renal function was defined as an eGFR $<60 \mathrm{ml} / \mathrm{min} / 1.73 \mathrm{~m}^{2}$ according to practice guidelines.

\subsection{Follow-up and ICD therapy event analysis}

Follow-up started at the time of CRT-D implantation. In Rotterdam, patients were seen at 2 weeks; at 3, and 6 months after implantation; and at 6 -month intervals thereafter. In Basel, patients were seen at 1 , 3 , and 6 months after implantation, and also at 6-month intervals thereafter. The programming of the ventricular tachycardia (VT) zone was set to $170-180 \mathrm{bpm}$. The ventricular fibrillation (VF) zone was set to 220-240 bpm. At each visit, arrhythmic events with stored electrograms (EGMs) were retrieved from the device's memory. Appropriate ICD therapy was defined as ICD therapy delivered for VT/VF. The presence of atrioventricular dissociation (ventricular rate $>$ atrial rate) was used to diagnose ventricular tachyarrhythmia when the baseline atrial rhythm is sinus rhythm. In case of atrial fibrillation as baseline atrial rhythm, ventricular tachyarrhythmias were defined as events with a sudden increase in rate combined with a change in ventricular near-field and far-field EGM morphology from the baseline rhythm without biventricular pacing.

\subsection{Endpoints}

The primary endpoint was all-cause mortality. Patients who underwent cardiac transplantation or left ventricular assist device (LVAD) implantation were censored at the time of surgery. The secondary endpoint was time from CRT-D implantation to either the occurrence of appropriate ICD shock within the VF zone of the device (being 'potentially life-threatening') or death without prior appropriate ICD shock (so-called 'prior death'). In the current study, death without prior appropriate ICD shock is the event of interest. The competing risk event is appropriate ICD shock. In case of tied events, the more serious event was always coded. If, for example, appropriate shock in the VF zone and death were tied on the same day, it was counted as death.

\subsection{Application of the Heart Failure Meta-score}

The predictive HF Meta-score is constructed of independent mortality predictors identified in a meta-analysis. These predictors include 3 continuous variables: age, LVEF and eGFR; a categoric variable: New York Heart Association (NYHA) functional class; and 11 dichotomous variables: male gender, African-American race, diabetes, chronic obstructive pulmonary disease (COPD), peripheral vascular disease (PVD), ischemic cardiomyopathy (iCMP), HF admission within 1 year prior to implantation, past or present atrial fibrillation, wide QRS ( $\geq 120 \mathrm{~ms}$ ), secondary prevention indication, and history of ICD shocks (appropriate and inappropriate). For all patients, the HF Meta-score was calculated using the mathematical formula as previously described by Alba et al. [11] As we evaluated primary prevention patients with a CRT-D in our study, the variable wide QRS ( $\geq 120 \mathrm{~ms}$ ) was answered 'yes', while secondary prevention indication and history of ICD shocks were answered 'no'.

\subsection{Statistical analysis}

Descriptive statistics are presented as mean and SD for continuous variables, if normally distributed, or as median and 25th and 75th percentiles. Data were compared with the Student's $t$-test or the MannWhitney $U$ test, where appropriate. Categorical data were expressed as percentages and compared with Fisher's exact test. Although most patients had a relatively complete dataset, the method of multiple 
imputation was used to include variables with $<5 \%$ of missing data; LVEF $3.5 \%$, creatinine $0.9 \%$, and NYHA $1.6 \%$.

The calculated HF Meta-score was converted to event-free survival probabilities up to 5 years for each individual patient (i.e. predicted survival). The observed survival rates were calculated according to the Kaplan-Meier method, and differences between risk quintiles were evaluated by the log-rank test. Discrimination was assessed by using the Harrell's C-statistic for time-to-event data. Model discrimination was deemed poor if the $C$-statistic was between 0.50 and 0.70 , good between 0.70 and 0.80 , and excellent if $>0.80$. Model calibration was visualized by plotting the predicted risks against the observed risks in a calibration-in-the-large plot stratifying by risk quintiles, and further described by the calibration slope and intercept. The intercept was used to assess whether the model predictions are systematically too low or too high. In case of perfect calibration, which means that the percentage predicted outcome is equal to the observed percentage, the intercept is 0 . If outcome rate is overestimated, the intercept is negative and vice versa. The calibration slope, which in an ideal case equals 1 , reflects how close the predicted average risk by quintiles is to the observed risk [13]. The HosmerLemeshow goodness-of-fit test was also used to assess calibration. As a measure of accuracy, the Brier score was calculated, which is the averaged squared difference between predicted and observed values. The Brier score ranges from 0 to 1 ; lower scores being better, a score of 0 indicates a perfect model. Usually, a model is considered useful if the Brier score is $<0.25$. To explore whether the HF Metascore adds to ICD decision making, competing risk analysis was performed. We used cumulative incidence functions (CIFs), which is the probability of occurrence of a particular event by time $t$ in the presence of competing events. The associated CIF plots were constructed. To analyze the effect of the HF Meta-score, the proportional subdistribution hazard model as proposed by Fine and Gray was used [14]. Subhazard ratios (SHRs) are presented with corresponding 95\% confidence intervals (CIs). Statistical analysis was performed using Stata version 16 SE for Windows (StataCorp, College Station, TX) and R statistical software, version 3.5.3. Statistical significance was defined as $P<0.05$ (two-tailed).

\section{Results}

The baseline characteristics of the study population are presented in Table 1. Briefly, median age of the study population was 66 years (range 58 to 72 years) and $76 \%$ were male. Sixty-six percent of patients were in NYHA functional class III or IV, mean LVEF was $25 \pm 6 \%$, and $47 \%$ percent had ischemic cardiomyopathy.

During a median follow-up of 5.2 years (range 2.3 to 8.7 years), 237 patients died, 8 underwent cardiac transplantation, and 5 underwent LVAD implantation. The mode of death was sudden, including arrhythmic, in $10 \%$, end-stage HF in $46 \%$, non-cardiac in $28 \%$, and unknown in $16 \%$. Overall, the annual mortality rate was 5.6\% (95\% CI 5.5\%-5.7\%), yielding a mortality rate of $6 \%, 18 \%$, and $37 \%$, at 1,3 , and 5 years, respectively. Mortality was not different between the different implant periods $(P=0.25)$. The Kaplan-Meier survival curves according to quintiles of the HF Meta-score estimated risk are shown in Fig. 1. At 5years follow-up, mortality ranged from $12 \%$ (95\% CI, 7\%-20\%) to $53 \%$ (95\% CI, 44\%-62\%), for quintiles 1 to 5 , respectively (overall log-rank $P<0.001)$. Mortality risk was increased with ascending quintiles of HF Meta-score, with a 6.9-fold higher mortality (HR 6.9; 95\% CI, 3.7-12.8) in the highest quintile compared to the lowest quintile (Table 2).

Model discrimination assessed by C-statistic was $0.76(95 \% \mathrm{CI}$, $0.71-0.82)$ at 1 year and $0.71(95 \% \mathrm{Cl}, 0.66-0.76)$ at 5 years. Model calibration was excellent as assessed by the calibration plot (Fig. 2), showing a calibration slope of 1 with an intercept of 0 indicating that the predicted risks were not over- or underestimated. No evidence of lack of fit was also assessed by the Hosmer-Lemeshow test (chi-square $=$ 3.68, $P=0.88$ ). The HF Meta-score appeared to be accurate based on the Brier score (value of 0.10 ) for predicting survival in patients with a CRT-D as primary prevention of SCD.

In Table 3, baseline characteristics in ascending quintiles of the HF Meta-score are presented. In the highest quintile, patients were significantly older and sicker compared to the lowest quintiles, as indicated by higher proportion of NYHA III-IV patients, more frequent ischemic cardiomyopathy and higher prevalence of comorbidity.

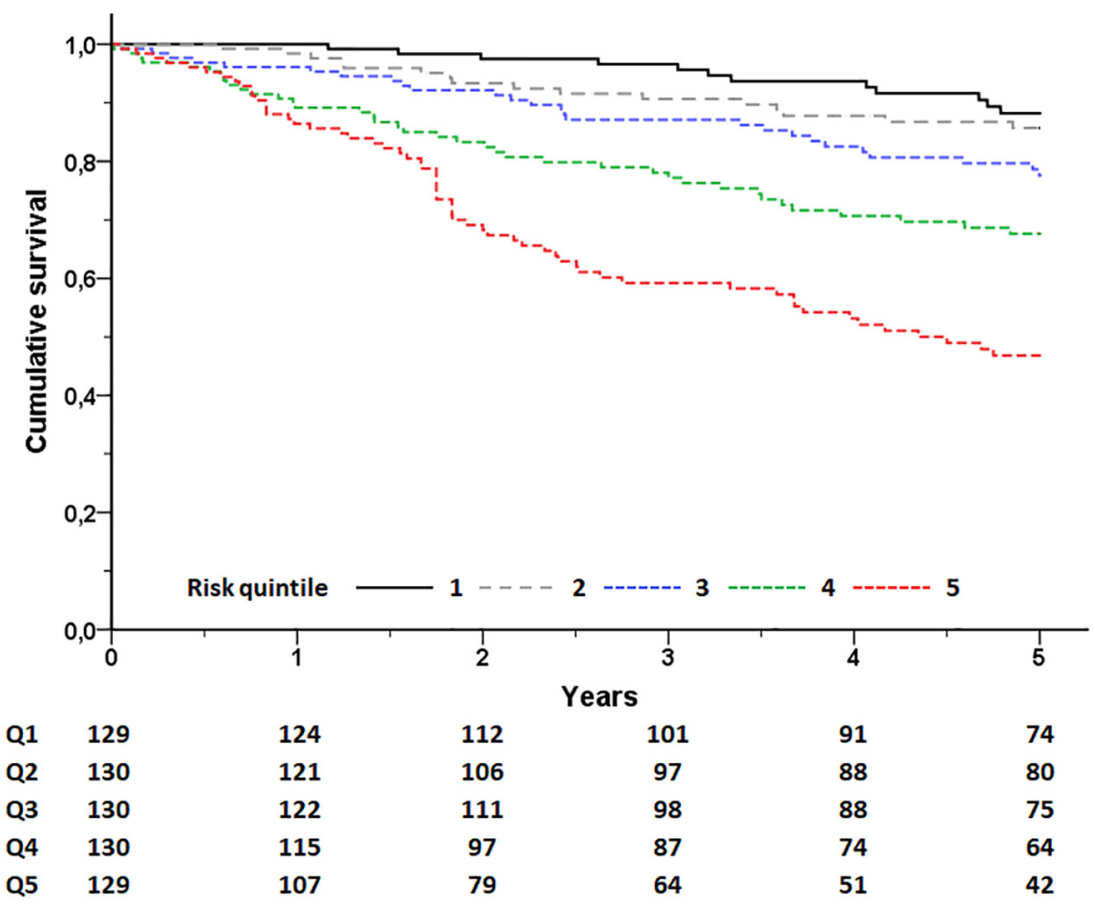

Fig. 1. Five-year survival by risk quintile predicted by the Heart Failure Meta-score. 
Table 2

All-cause mortality by quintiles of HF Meta-score predicted risk.

\begin{tabular}{lllll}
\hline Quintile & HF Meta-score & Total deaths & HR $(95 \% \mathrm{CI})$ & $P$-value \\
\hline I $(\mathrm{n}=129)$ & $0.64-1.75$ & $12(9 \%)$ & Reference & Reference \\
II $(\mathrm{n}=130)$ & $1.75-2.16$ & $16(12 \%)$ & $1.4(0.6-2.9)$ & 0.41 \\
III $(\mathrm{n}=130)$ & $2.16-2.59$ & $26(20 \%)$ & $2.2(1.1-4.4)$ & 0.023 \\
IV $(\mathrm{n}=130)$ & $2.59-3.05$ & $38(29 \%)$ & $3.6(1.9-6.9)$ & $<0.001$ \\
V $(\mathrm{n}=129)$ & $3.05-6.17$ & $60(47 \%)$ & $6.9(3.7-12.8)$ & $<0.001$ \\
\hline
\end{tabular}

At 5-years follow-up, 51 patients (8\%) experienced an appropriate ICD shock. The five-year cumulative incidence of appropriate ICD shock was $4 \%$ in the lowest quintile and $12 \%$ in the highest quintile. A total of 180 patients (28\%) reached the secondary endpoint of time to first appropriate ICD shock or death without prior appropriate ICD shock. Of these patients, death without prior appropriate ICD shock was observed in 129 patients (72\%). In Fig. 3, the cumulative incidence curves of death without prior appropriate ICD shock per quintile of the

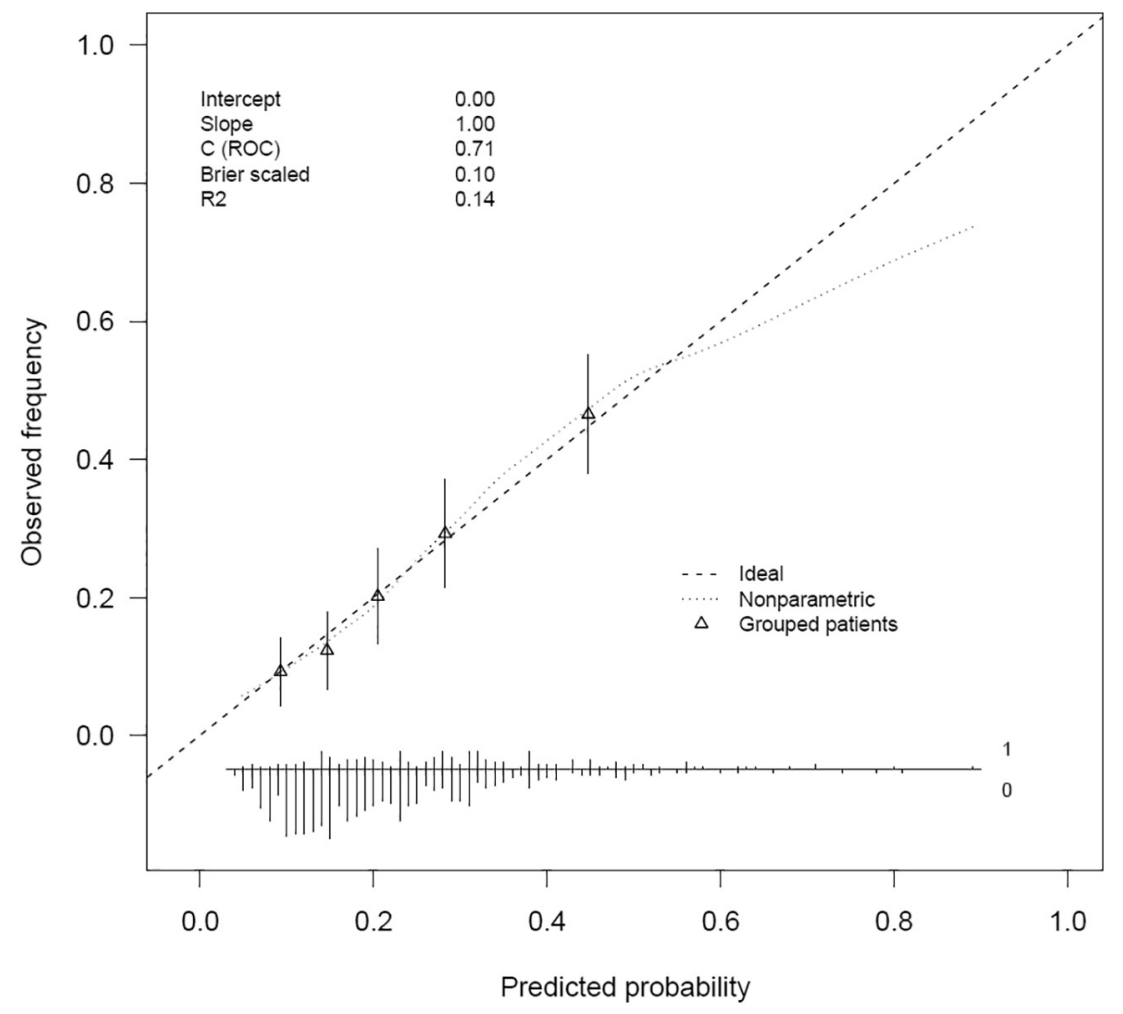

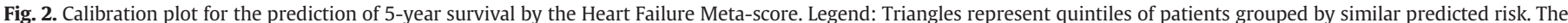
distribution of patients is indicated with spikes at the bottom of the graph, stratified by endpoint (death above the X-axis, survivors below the x-axis).

Table 3

Baseline characteristics by HF Meta-score subgroups.

\begin{tabular}{|c|c|c|c|c|c|c|}
\hline Variable & $\begin{array}{l}\text { Quintile } 1 \\
(\mathrm{n}=129)\end{array}$ & $\begin{array}{l}\text { Quintile 2 } \\
(\mathrm{n}=130)\end{array}$ & $\begin{array}{l}\text { Quintile } 3 \\
(\mathrm{n}=130)\end{array}$ & $\begin{array}{l}\text { Quintile4 } \\
(\mathrm{n}=130)\end{array}$ & $\begin{array}{l}\text { Quintile } 5 \\
(\mathrm{n}=129)\end{array}$ & $P$-value \\
\hline Age (y) & $56(50-62)$ & $63(55-68)$ & $67(61-71)$ & $71(65-75)$ & $72(67-77)$ & $<0.001$ \\
\hline Male gender & $90(70 \%)$ & $94(72 \%)$ & $97(75 \%)$ & $108(83 \%)$ & $103(80 \%)$ & 0.08 \\
\hline Atrial fibrillation & $2(2 \%)$ & $17(13 \%)$ & $19(15 \%)$ & $34(26 \%)$ & $53(41 \%)$ & $<0.001$ \\
\hline Ischemic cardiomyopathy & $25(19 \%)$ & $44(34 \%)$ & $67(52 \%)$ & $79(61 \%)$ & $90(70 \%)$ & $<0.001$ \\
\hline \multicolumn{7}{|l|}{ NYHA functional class } \\
\hline II & $92(71 \%)$ & $58(45 \%)$ & $31(24 \%)$ & $26(20 \%)$ & $12(9 \%)$ & \\
\hline III & 37 (29\%) & $72(55 \%)$ & $99(76 \%)$ & $101(79 \%)$ & $96(74 \%)$ & $<0.001$ \\
\hline IV & $0(0 \%)$ & $0(0 \%)$ & $0(0 \%)$ & $1(1 \%)$ & $21(16 \%)$ & \\
\hline $\operatorname{LVEF}(\%)$ & $27 \pm 6$ & $25 \pm 6$ & $25 \pm 6$ & $24 \pm 6$ & $24 \pm 6$ & $<0.001$ \\
\hline QRS duration $(\mathrm{ms})$ & $169 \pm 22$ & $166 \pm 23$ & $168 \pm 21$ & $163 \pm 22$ & $168 \pm 27$ & 0.37 \\
\hline Heart failure admission 1 year prior to implantation & $6(5 \%)$ & $23(25 \%)$ & $39(30 \%)$ & $46(35 \%)$ & $84(65 \%)$ & $<0.001$ \\
\hline \multicolumn{7}{|l|}{ Comorbidities } \\
\hline Diabetes mellitus & $7(5 \%)$ & $13(10 \%)$ & $31(24 \%)$ & $51(39 \%)$ & $61(47 \%)$ & $<0.001$ \\
\hline COPD & $5(4 \%)$ & $8(6 \%)$ & $10(8 \%)$ & $21(16 \%)$ & $41(32 \%)$ & $<0.001$ \\
\hline Peripheral vascular disease & $3(2 \%)$ & $4(3 \%)$ & $6(5 \%)$ & $12(9 \%)$ & $28(22 \%)$ & $<0.001$ \\
\hline \multicolumn{7}{|l|}{ Laboratory data } \\
\hline Sodium $(\mathrm{mmol} / \mathrm{l})$ & $140 \pm 3$ & $140 \pm 3$ & $139 \pm 4$ & $139 \pm 5$ & $138 \pm 4$ & $<0.001$ \\
\hline Hemoglobin (g/dl) & $14.3 \pm 1.5$ & $13.9 \pm 1.6$ & $13.7 \pm 1.7$ & $13.3 \pm 1.9$ & $13.0 \pm 1.8$ & $<0.001$ \\
\hline Creatinine $(\mathrm{mg} / \mathrm{dl})$ & $0.9(0.8-1.1)$ & $1.0(0.9-1.2)$ & $1.2(1.0-1.4)$ & $1.3(1.0-1.6)$ & $1.6(1.2-2.0)$ & $<0.001$ \\
\hline $\mathrm{GFR}\left(\mathrm{ml} / \mathrm{min} / 1.73 \mathrm{~m}^{2}\right)$ & $81(68-93)$ & $71(58-89)$ & $60(48-78)$ & $56(44-68)$ & $42(32-60)$ & $<0.001$ \\
\hline $\mathrm{GFR}<60 \mathrm{ml} / \mathrm{min} / 1.73 \mathrm{~m}^{2}$ & $18(14 \%)$ & $37(29 \%)$ & $64(49 \%)$ & $79(61 \%)$ & $96(74 \%)$ & $<0.001$ \\
\hline
\end{tabular}

Continuous data are expressed as mean \pm SD or median (interquartile range), and categorical data as number (percentage).

COPD, chronic obstructive pulmonary disease; GFR, glomerular filtration rate; LVEF, left ventricular ejection fraction; NYHA, New York Heart Association. 


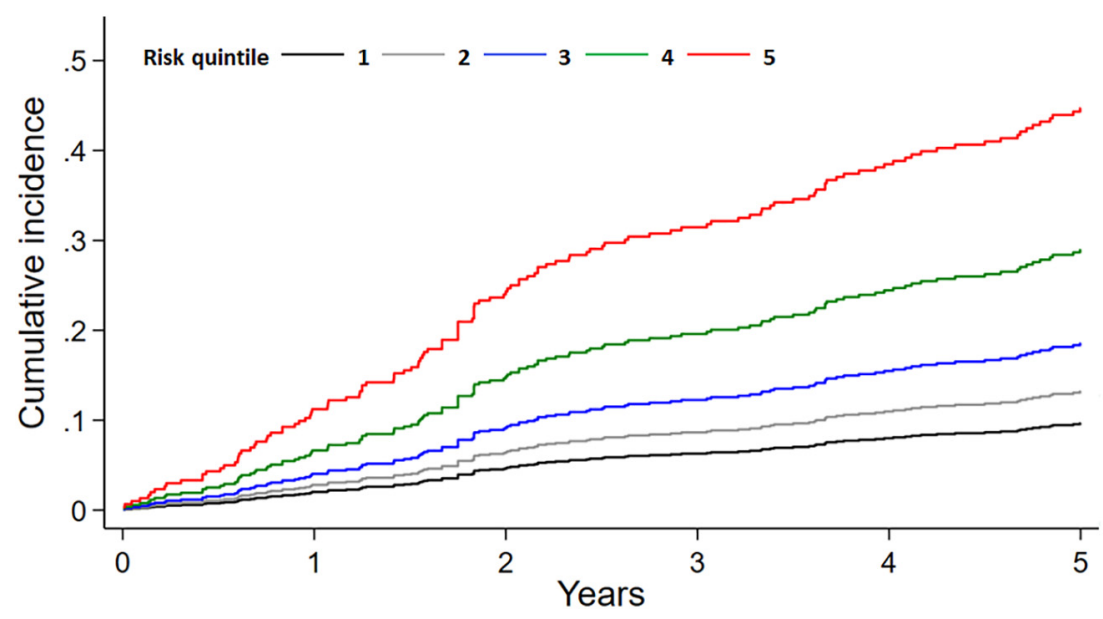

Fig. 3. The 5-year cumulative incidence curves of mortality without prior appropriate ICD shock per risk quintile of the Heart Failure Meta-score.

HF Meta-score are presented. Death without prior appropriate ICD shock was almost 6 times higher for patients in the highest risk quintile compared to the lowest risk quintile (SHR 5.8; 95\% CI 3.1-11.0, $P<0.001)$.

\section{Discussion}

In the present study, we evaluated the performance of the HF Meta-score in patients with HF who received CRT-D devices for primary prevention of SCD. The main findings of our study are that (1) the calibration of the HF Meta-score is excellent for prediction of survival in patients with a CRT-D, (2) the ability of the HF Meta-score to discriminate between patients with low or high risk for mortality was good, and (3) patients in the highest risk quintile were 5.8 more likely to die without ICD shock compared to patients in the lowest risk quintile.

Heart failure is a major cause of cardiovascular mortality and morbidity, and its prevalence and incidence are still increasing [1]. In patients with HFrEF, NYHA class $\geq \mathrm{II}$, and prolonged QRS duration, CRT improves clinical symptoms, reduces hospitalizations and prolongs survival. The majority of patients eligible for CRT also qualify for defibrillator therapy as primary prevention of SCD. Based on this, current guidelines advocate to implant a CRT-D in eligible patients [7,8]. Although CRT-D therapy has been shown to decrease mortality and HF events, the benefit of CRT-D is not uniform among patients. Accurately predicting prognosis is important to identify those patients who will likely not benefit from ICD back-up, and would be ipso facto candidates for a CRT pacemaker. However, prediction of prognosis in patients with HFrEF is challenging due to the increasing proportion of elderly patients who present with multiple comorbidities. To overcome this problem, some investigators have developed predictive models with the intent to identify patients who are at high risk for all-cause mortality and who are therefore less likely to benefit from defibrillator therapy. The SHFM was originally developed in the late 1990s when only a minority of patients received HF therapy according to current practice [10]. Since its introduction, the SHFM underwent several modifications [15] and has been validated in cohorts of patients treated with CRT-D. C-statistics ranged between 0.65 and 0.75 over a follow-up of 5 years [16-18].

Goldenberg et al. developed a score constructed of five risk factors to assess benefit from ICD therapy based on the MADIT-II study population consisting of patients with a previous myocardial infarction and LVEF $\leq 30 \%$ [19]. In a post hoc analysis of MADIT-II cohort, patients with $0-2$ risk factors had a clear survival benefit from the ICD, whereas patients with $\geq 3$ risk factors had not [19]. Barra et al. evaluated the MADIT-II score in patients treated with CRT [20]. They found similar results: benefit from a CRT-D decreased with increasing number of risk factors. However, the prognostic performance of the MADIT IIscore is limited, C-statistics range between 0.61 and 0.71 .

Previous studies have shown the substantial risk of mortality in patients with an ICD who have concomitant non-cardiac comorbidities [21-24]. A recent meta-analysis of 4 major randomized clinical trials evaluating the survival benefit of primary prevention ICDs demonstrated that patients with extensive concomitant comorbidity may experience less benefit from ICD than those with less comorbidity [25]. In particular, diabetes, COPD, PVD, impaired renal function and AF are independently associated with increased risk of mortality [24]. The impact of comorbidities is commonly evaluated by using the Charlson comorbidity index [22-24]. Data from an Italian registry found that patient age and a higher NYHA functional class in addition to comorbidities are also important factors affecting patient outcome [23]. Prognosis in HF is affected by a constellation of multiple factors. Using one factor (e.g. age or NYHA class) or only a small number of factors (e.g. the Charlson comorbidity index) will lead to suboptimal shared decision makings. For example, an old patient may have better prognosis compared to a NYHA class IV patient with impaired kidney function. The use of scores incorporating multiple factors with an impact on prognosis most probably result in better patient selection or counseling.

In the current study, we evaluated the performance of the HF Metascore based on patient-level data including significant comorbidities, age, LVEF and NYHA class, in order to predict prognosis [11]. The performance of the HF Meta-score was good in predicting short- and long-term survival, with $C$-statistics ranging between 0.76 at 1 -year follow-up and 0.71 at 5-years follow-up. The HF Meta-score showed excellent calibration and accuracy. We found that increasing mortality risk at the time of CRT implant was associated with less benefit from the ICD as defined by the occurrence of death without receiving an appropriate ICD shock. When looking at clinical variables, patients at high risk for mortality were older, had more ischemic cardiomyopathy, and a high number of comorbidities. These results pose uncertainty regarding the utilization of a CRT-D in high risk patients in whom a CRT pacemaker (CRT-P) may be a more appropriate approach.

In the landmark COMPANION trial, the difference in all-cause mortality between CRT-D and CRT-P was not tested [4]. A few studies have attempted to compare the outcomes between CRT-P and CRT-D patients [26,27]. A large multicenter prospective registry, CeRtiTuDe, evaluated the characteristics of CRT-P versus CRT-D patients in a realworld scenario [28]. Cause-of-death analysis demonstrated that excess mortality among CRT-P patients was almost entirely related to nonSCD. The CRT-P patients were older, had more advanced heart failure and co-morbidities when compared to CRT-D patients. This finding 
was also confirmed in a meta-analysis to determine the importance of ICD back-up in CRT recipients [29]. Patients with ischemic cardiomyopathy seemed to benefit from the addition of the ICD, while such benefit was less clear in those with non-ischemic cardiomyopathy. Another relevant finding was that patients with non-ischemic cardiomyopathy receiving CRT-P were in general older, had more advanced heart failure and a higher number of comorbidities compared to non-ischemic CRTD patients. Taking everything together, the use of the HF Meta-score may assist physicians in the shared decision-making process with HF patients eligible for CRT-D as primary prevention of SCD. In a subgroup of these patients, competing risks of non-sudden death may diminish the value of ICD back-up to CRT.

\subsection{Limitations}

The present study had some limitations. First, this observational study is limited by the non-randomized design. However, the results of this study are representative for patients who received a CRT-D as primary prevention in routine clinical practice. Future changes in therapy and management strategies might improve patient outcomes and potentially change the association between predictors and outcomes. In general, the performance of risk models should be retested routinely in order to determine whether discrimination is still adequate. The study cohort included patients over a 12-year period, during which treatment of HF changed. In the same period, the programming of devices with respect to detection and treatment of ventricular arrhythmias changed. We accounted for this by defining three groups according to the date of implant, where no difference was noticed.

A recent model the Seattle Proportional Risk Models (SPRM) has been developed to estimate the proportion of total mortality due to sudden death in order to determine ICD benefit [30]. The HF Meta-score has been developed to predict the risk of all-cause mortality and not specifically causes of death as in the SPRM. Comparison of the HF Meta-score with the SPRM is beyond the scope of the current study.

\subsection{Conclusion}

The present study found that the HF Meta-score has a very good performance to predict survival in HF patients treated at the time of CRT-D implantation as primary prevention of SCD. The HF Meta-score proved to be useful in identifying a subgroup of patients with poor prognosis and high probability of death prior to receiving ICD shock therapy, favoring the sole implant of CRT. The use of the HF Meta-score may assist physicians in a more personalized shared decision-making process of HF patients with different prognosis who are potentially eligible for CRT-D as primary prevention of SCD.

\section{Author agreement form}

Manuscript Title: Application of the Heart Failure Meta-score to Predict Prognosis in Patients with Cardiac Resynchronization Defibrillators.

List of all Authors: Dominic A.M.J. Theuns, PhD, Beat A. Schaer, MD, Kadir Caliskan, MD, PhD, Sanne E. Hoeks, PhD, Christian Sticherling, MD, Sing-Chien Yap MD, PhD, Ana C. Alba MD, PhD.

Corresponding Author: Dominic A.M.J. Theuns, PhD.

This statement is to certify that all authors have seen and approved the manuscript.

being submitted, have contributed significantly to the work, attest to the validity and legitimacy of the data and its interpretation, and agree to its submission to the International Journal of Cardiology.

We attest that the article is the Authors' original work, has not received prior publication and is not under consideration for publication elsewhere. We adhere to the statement of ethical publishing as appears in the International of Cardiology (citable as: Shewan LG, Rosano GMC, Henein MY, Coats AJS. A statement on ethical standards in publishing scientific articles in the International Journal of Cardiology family of journals. Int. J. Cardiol. 170 (2014) 253-254 DOI:https://doi.org/10. 1016/j.ijcard.2013.11).

On behalf of all Co-Authors, the corresponding Author shall bear full responsibility for the submission. Any changes to the list of authors, including changes in order, additions or removals will require the submission of a new author agreement form approved and signed by all the original and added submitting authors.

All authors are requested to disclose any actual or potential conflict of interest including any financial, personal or other relationships with other people or organizations within three years of beginning the submitted work that could inappropriately influence, or be perceived to influence, their work. If there are no conflicts of interest, the COI should read: "The authors report no relationships that could be construed as a conflict of interest".

\section{Declaration of Competing Interest}

Dr. Theuns has received research grants from Biotronik and Boston Scientific and consulting fees from Boston Scientific. Dr. Schaer is listed on the speaker's bureau for Medtronic. Dr. Sticherling has received speaker fees from Boston Scientific, Biotronik and Microport CRM and consulting fees from Biotronik, Boston Scientific, and Medtronic. Dr. Yap is listed as an ad hoc consultant for Medtronic and Boston Scientific and has been a speaker for Medtronic. The other authors have nothing to disclose.

\section{References}

[1] A.P. Ambrosy, G.C. Fonarow, J. Butler, O. Chioncel, S.J. Greene, M. Vaduganathan, et al., The global health and economic burden of hospitalizations for heart failure: lessons learned from hospitalized heart failure registries, J. Am. Coll. Cardiol. 63 (2014) 1123-1133.

[2] P.A. McCullough, E.F. Philbin, J.A. Spertus, S. Kaatz, K.R. Sandberg, W.D. Weaver, et al., Confirmation of a heart failure epidemic: findings from the resource utilization among congestive heart failure (REACH) study, J. Am. Coll. Cardiol. 39 (2002) 60-69.

[3] W.T. Abraham, W.G. Fisher, A.L. Smith, D.B. Delurgio, A.R. Leon, E. Loh, et al., Cardiac resynchronization in chronic heart failure, N. Engl. J. Med. 346 (2002) 1845-1853.

[4] M.R. Bristow, L.A. Saxon, J. Boehmer, S. Krueger, D.A. Kass, T. De Marco, et al. Cardiac-resynchronization therapy with or without an implantable defibrillator in advanced chronic heart failure, N. Engl. J. Med. 350 (2004) 2140-2150.

[5] J.G. Cleland, J.C. Daubert, E. Erdmann, N. Freemantle, D. Gras, L. Kappenberger, et al., The effect of cardiac resynchronization on morbidity and mortality in heart failure, N. Engl. J. Med. 352 (2005) 1539-1549.

[6] A.J. Moss, W.J. Hall, D.S. Cannom, H. Klein, M.W. Brown, J.P. Daubert, et al., Cardiacresynchronization therapy for the prevention of heart-failure events, N. Engl. J. Med. 361 (2009) 1329-1338.

[7] C.W. Yancy, M. Jessup, B. Bozkurt, J. Butler, D.E. Casey Jr., M.H. Drazner, et al., 2013 ACCF/AHA guideline for the management of heart failure: a report of the American College of Cardiology Foundation/American Heart Association Task Force on Practice Guidelines, J. Am. Coll. Cardiol. 62 (2013) e147-e239.

[8] P. Ponikowski, A.A. Voors, S.D. Anker, H. Bueno, J.G. Cleland, A.J. Coats, et al., 2016 ESC Guidelines for the diagnosis and treatment of acute and chronic heart failure: The Task Force for the diagnosis and treatment of acute and chronic heart failure of the European Society of Cardiology (ESC)Developed with the special contribution of the Heart Failure Association (HFA) of the ESC, Eur. Heart J. 37 (2016) 2129-2200.

[9] K.D. Aaronson, J.S. Schwartz, T.M. Chen, K.L. Wong, J.E. Goin, D.M. Mancini, Development and prospective validation of a clinical index to predict survival in ambulatory patients referred for cardiac transplant evaluation, Circulation 95 (1997) 2660-2667.

[10] W.C. Levy, D. Mozaffarian, D.T. Linker, S.C. Sutradhar, S.D. Anker, A.B. Cropp, et al., The Seattle Heart Failure Model: prediction of survival in heart failure, Circulation 113 (2006) 1424-1433.

[11] A.C. Alba, S.D. Walter, G.H. Guyatt, W.C. Levy, J. Fang, H.J. Ross, et al., Predicting survival in heart failure patients with implantable cardioverter-defibrillators: the heart failure meta-score, J. Card. Fail. 24 (2018) 735-745.

[12] K/DOQI, clinical practice guidelines for chronic kidney disease: evaluation, classification, and stratification, Am. J. Kidney Dis. 39 (2002) S1-266.

[13] E.W. Steyerberg, Y. Vergouwe, Towards better clinical prediction models: seven steps for development and an ABCD for validation, Eur. Heart J. 35 (2014) 1925-1931.

[14] J.P. Fine, R.J. Gray, A proportional hazards model for the subdistribution of a competing risk, J. Am. Stat. Assoc. 94 (1999) 496-509.

[15] W.C. Levy, K.L. Lee, A.S. Hellkamp, J.E. Poole, D. Mozaffarian, D.T. Linker, et al., Maximizing survival benefit with primary prevention implantable cardioverterdefibrillator therapy in a heart failure population, Circulation 120 (2009) 835-842. 
[16] M. Clemens, Z. Szegedi, L. Kardos, E. Nagy-Balo, G. Sandorfi, I. Edes, et al., The Seattle Heart Failure Model predicts survival in patients with cardiac resynchronization therapy: a validation study, J. Card. Fail. 18 (2012) 682-687.

[17] F. Regoli, F. Scopigni, F. Leyva, M. Landolina, S. Ghio, M. Tritto, et al., Validation of Seattle Heart Failure Model for mortality risk prediction in patients treated with cardiac resynchronization therapy, Eur. J. Heart Fail. 15 (2013) 211-220.

[18] T. Smith, W.C. Levy, B.A. Schaer, A.H. Balk, C. Sticherling, L. Jordaens, et al., Performance of the Seattle Heart Failure Model in implantable defibrillator patients treated with cardiac resynchronization therapy, Am. J. Cardiol. 110 (2012) 398-402.

[19] I. Goldenberg, A.K. Vyas, W.J. Hall, A.J. Moss, H. Wang, H. He, et al., Risk stratification for primary implantation of a cardioverter-defibrillator in patients with ischemic left ventricular dysfunction, J. Am. Coll. Cardiol. 51 (2008) 288-296.

[20] S. Barra, K.L. Looi, P.R. Gajendragadkar, F.Z. Khan, M. Virdee, S. Agarwal, Applicability of a risk score for prediction of the long-term benefit of the implantable cardioverter defibrillator in patients receiving cardiac resynchronization therapy, Europace 18 (2016) 1187-1193.

[21] D.S. Lee, J.V. Tu, P.C. Austin, P. Dorian, R. Yee, A. Chong, et al., Effect of cardiac and noncardiac conditions on survival after defibrillator implantation, J. Am. Coll. Cardiol. 49 (2007) 2408-2415.

[22] D.A. Theuns, B.A. Schaer, O.I. Soliman, D. Altmann, C. Sticherling, M.L. Geleijnse, et al., The prognosis of implantable defibrillator patients treated with cardiac resynchronization therapy: comorbidity burden as predictor of mortality, Europace 13 (2011) 62-69.

[23] G. Boriani, E. Berti, L.M. Belotti, M. Biffi, R. De Palma, V.L. Malavasi, et al., Cardiac device therapy in patients with left ventricular dysfunction and heart failure: 'realworld' data on long-term outcomes (mortality, hospitalizations, days alive and out of hospital), Eur. J. Heart Fail. 18 (2016) 693-702.

[24] A.C. Ruwald, M. Vinther, G.H. Gislason, J.B. Johansen, J.C. Nielsen, H.H. Petersen, et al., The impact of co-morbidity burden on appropriate implantable cardioverter defibrillator therapy and all-cause mortality: insight from Danish nationwide clinical registers, Eur. J. Heart Fail. 19 (2017) 377-386.

[25] B.A. Steinberg, S.M. Al-Khatib, R. Edwards, J. Han, G.H. Bardy, J.T. Bigger, et al., Outcomes of implantable cardioverter-defibrillator use in patients with comorbidities: results from a combined analysis of 4 randomized clinical trials, JACC Heart Fail. 2 (2014) 623-629.

[26] K.L. Looi, P.R. Gajendragadkar, F.Z. Khan, M. Elsik, D.A. Begley, S.P. Fynn, et al., Cardiac resynchronisation therapy: pacemaker versus internal cardioverterdefibrillator in patients with impaired left ventricular function, Heart 100 (2014) 794-799.

[27] G. Morani, M. Gasparini, F. Zanon, E. Casali, A. Spotti, A. Reggiani, et al., Cardiac resynchronization therapy-defibrillator improves long-term survival compared with cardiac resynchronization therapy-pacemaker in patients with a class IA indication for cardiac resynchronization therapy: data from the Contak Italian registry, Europace 15 (2013) 1273-1279.

[28] E. Marijon, C. Leclercq, K. Narayanan, S. Boveda, D. Klug, J. Lacaze-Gadonneix, et al., Causes-of-death analysis of patients with cardiac resynchronization therapy: an analysis of the CeRtiTuDe cohort study, Eur. Heart J. 36 (2015) 2767-2776.

[29] S. Barra, R. Providencia, A. Tang, P. Heck, M. Virdee, S. Agarwal, Importance of implantable cardioverter-defibrillator Back-up in cardiac resynchronization therapy recipients: a systematic review and meta-analysis, J. Am. Heart Assoc. 4 (2015) https://doi.org/10.1161/JAHA.115.002539.

[30] R. Shadman, J.E. Poole, T.F. Dardas, D. Mozaffarian, J.G. Cleland, K. Swedberg, et al., A novel method to predict the proportional risk of sudden cardiac death in heart failure: derivation of the Seattle proportional risk model, Heart Rhythm 12 (2015) 2069-2077. 